\title{
NAME OF THE REVIEWERS OF ARTICLES IN THIS ISSUE
}

(J Bangladesh Coll Phys Surg 2010; 28: 68)

Dr. Ferdousi Begum \& Dr. Fahmida Zabin

Professor U.H. Shahera Khatun \& Professor M.A. Salam

Prof. Sabera Khatun \& Dr. Fahmida Zabin

Dr. Mizanur Ragman \& Dr. Abdul Waded Chowdhury

Prof. Md. Moyeenuzzaman, Prof. Md. Ismail Patwary \& Dr. Minhaj R Chowdhery

Prof. Mst. Sabera KHaitian \& Dr. Laila Parveen Banu

Prof. Farhana Dewan \& Dr. Fatema

Prof Md. Ismail Patary \& Dr. (Lt. Col.) Mamun Mostafi

Prof. Nazmul Alan \& Dr. Ahmed Murtuza

Prof. Ekhlasur Rahman \& Prof. Choudhury Ali Kawser 